\title{
Pseudopotentials from electron density
}

\author{
Á. Nagy and I. Andrejkovics \\ Institute of Theoretical Physics, Kossuth Lajos University, H-4010 Debrecen, Hungary
}

(Received 7 March 1995; revised manuscript received 13 November 1995)

\begin{abstract}
A method is introduced that allows the construction of pseudopotentials in the density-functional theory. This method is based on a procedure worked out by one of the authors [J. Phys. B 26, 43 (1993); Philos. Mag. B 69, 779 (1994)] for determining Kohn-Sham potentials, one-electron orbitals, and energies from the electron density. The Hartree-Fock densities of Bunge, Barrientos, and Bunge [At. Data Nucl. Data Tables 53, 114 (1993)] are used to obtain the Kohn-Sham potentials of the Li, Na, and K atoms, and then Phillips-Kleinmantype [Phys. Rev. 116, 287 (1959); 118, 1153 (1960)] pseudopotentials are calculated. The arbitrariness of the pseudo-orbital is removed by minimization of the kinetic energy.
\end{abstract}

PACS number(s): 31.15.Ew, 71.10.-w

\section{INTRODUCTION}

Pseudopotentials are widely used for a large variety of problems. Recently, pseudopotentials have been introduced into the density-functional theory [1], too. These are based on the local density approximation (LDA).

The problem of obtaining the Kohn-Sham potential with the knowledge of the electron density has become of considerable interest $[2,3]$. Several methods have already been proposed to solve this question [4-9]. One of the authors has also presented such a method for determining Kohn-Sham and exchange potentials in the exchange-only densityfunctional theory [5]. Here, pseudopotentials determined by this method are presented. These are exact pseudopotentials of Phillips-Kleinman type [10].

\section{KOHN-SHAM POTENTIAL IN THE DENSITY-FUNCTIONAL THEORY}

First, the method obtaining Kohn-Sham potential $v_{\mathrm{KS}}$, one-electron orbitals $u_{i}$, and energies $\varepsilon_{i}$ is summarized [5]. Usually the Kohn-Sham equations

$$
\left[-\frac{1}{2} \nabla^{2}+v_{\mathrm{KS}}(\mathbf{r})\right] u_{i}(\mathbf{r})=\varepsilon_{i} u_{i}(\mathbf{r})
$$

are solved self-consistently using some kind of approximation for the exchange-correlation potential $v_{\mathrm{xc}}$. If we know the density, however, we can solve the inverse problem, i.e., we can determine the Kohn-Sham potential. A possible solution to this problem is the following. Starting out from an approximate potential the Kohn-Sham equations are solved and the density $n^{(1)}$ of the first iteration is determined. Then the Kohn-Sham potential of the second iteration is constructed and the Kohn-Sham equations are solved again. The process goes on until the density equals the input density.

If the process converges it converges to the true KohnSham potential corresponding to the input density because of the Hohenberg-Kohn theorem. It was found that the starting potential can be constructed using a simple LDA (e.g., $X \alpha$ ) potential. The Kohn-Sham potential in the $(i+1)$ th iteration

$$
v_{\mathrm{KS}}^{(i+1)}=\left[\gamma \frac{\varrho^{(i)}}{\varrho_{\text {input }}}+(1-\gamma)\right] v_{\mathrm{KS}}^{(i)}
$$

is obtained from the potential and density of the $i$ th iteration. This scheme with $\gamma=0.6$ leads to an appropriate convergence.

From the Kohn-Sham potential the exchange-correlation potential can be readily obtained. If the exact density is known, this procedure makes it possible to determine the Kohn-Sham potential exactly. If, however, only the HartreeFock density is available, one can obtain a very accurate exchange potential. This is not the exact exchange potential of the density-functional theory, though, because the Hartree-Fock density does not equal exactly the exchangeonly density functional density. This question is detailed in two recent papers $[11,12]$. It was found $[11,12]$ that the relative difference in the exchange energy is about $10^{-4}$,

$$
\left|\frac{E_{x}^{\mathrm{HF}}[\varrho]-E_{x}^{\mathrm{KS}}[\varrho]}{E_{x}^{\mathrm{HF}}[\varrho]}\right|<4.4 \times 10^{-4} .
$$

Earlier [5] it was demonstrated that

$$
\left|\frac{E_{x}^{\mathrm{HF}}[\varrho]-E_{x}^{\mathrm{KS}}\left[\varrho_{\mathrm{HF}}\right]}{E_{x}^{\mathrm{HF}}[\varrho]}\right|<1.5 \times 10^{-3} .
$$

So, though the exchange potential and energy obtained from the Hartree-Fock density are not the exact exchange potential and energy, they are very close to the exact ones.

\section{CONSTRUCTION OF PSEUDOPOTENTIALS}

With the knowledge of the Kohn-Sham one-electron orbitals and energies, pseudopotentials can be constructed. The

TABLE I. The pseudopotential coefficients of the Li, Na, and $\mathrm{K}$ atoms calculated by ourselves and Szasz and McGinn.

\begin{tabular}{lccccccc}
\hline \hline & \multicolumn{3}{c}{ Our results } & & \multicolumn{3}{c}{ Results of Szasz and McGinn } \\
\cline { 2 - 4 } \cline { 7 - 8 } Atom & $1 s$ & $2 s$ & $3 s$ & & $1 s$ & $2 s$ & $3 s$ \\
\hline $\mathrm{Li}$ & 0.2072 & & & & 0.1658 & & \\
$\mathrm{Na}$ & 0.0237 & 0.2443 & & & 0.0214 & -0.2193 & \\
$\mathrm{~K}$ & 0.0075 & 0.0617 & 0.3298 & & 0.0052 & 0.0544 & 0.3022 \\
\hline \hline
\end{tabular}




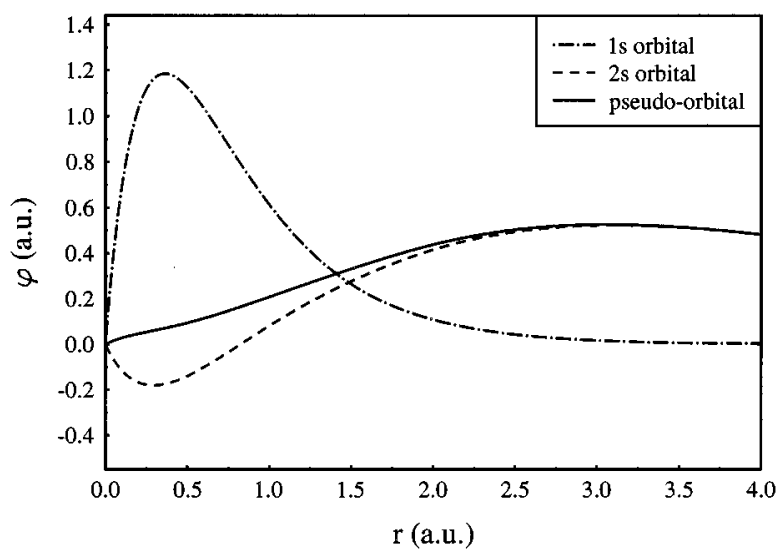

FIG. 1. Orbitals and the pseudo-orbital of the Li atom.

pseudopotentials are obtained using the method of Phillips and Kleinman [10].

Let us treat a system with one valence electron only. The Kohn-Sham core orbitals are denoted by $u_{1}, u_{2}, \ldots, u_{n}$. The valence orbital $\varphi$ is given by

$$
\varphi=\psi-\sum_{i=1}^{N} \alpha_{i} u_{i}
$$

where $\psi$ is the pseudo-orbital and $\alpha_{i}=\left\langle u_{i} \mid \psi\right\rangle$. Substituting $\varphi$ into the Kohn-Sham equation for the valence electron

$$
\left(-\frac{1}{2} \nabla^{2}+v_{\mathrm{KS}}\right) \varphi=\varepsilon \varphi
$$

we obtain the following equation for $\psi$ :

$$
\left(-\frac{1}{2} \nabla^{2}+v_{\mathrm{KS}}+v_{p}\right) \psi=\varepsilon \psi
$$

The local Phillips-Kleinman pseudopotential $v_{p}$ is given by

$$
v_{p}=\sum_{i=1}^{N} \alpha_{i} \frac{\left(\varepsilon-\varepsilon_{i}\right) u_{i}}{\psi} .
$$

It was observed by Cohen and Heine [13] that the definition of the pseudopotential is not uniquely determined. They showed that the pseudo-orbital can be subjected to an addi-

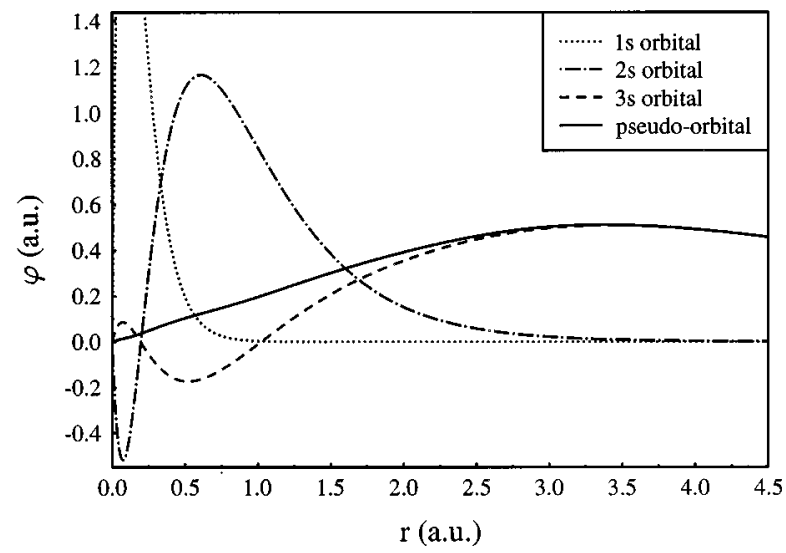

FIG. 2. Orbitals and the pseudo-orbital of the $\mathrm{Na}$ atom.

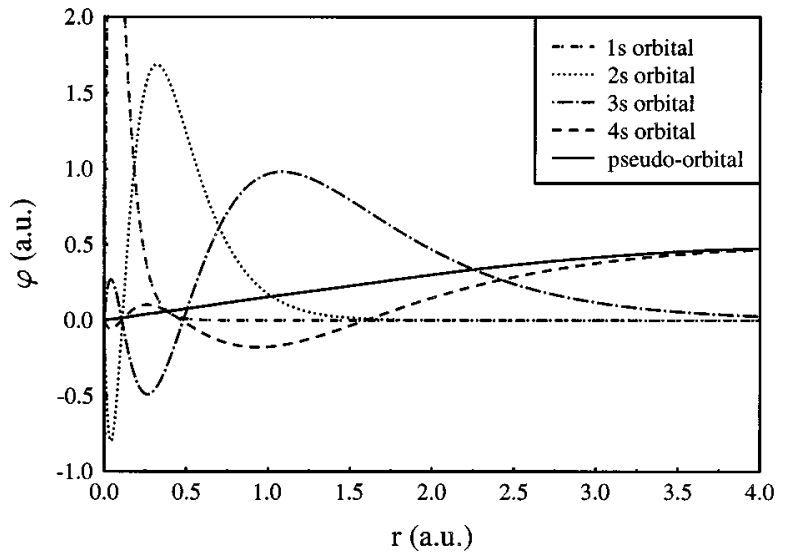

FIG. 3. Orbitals and the pseudo-orbital of the $\mathrm{K}$ atom.

tional requirement that does not disturb the exactness of the theory. The most plausible additional requirement, suggested also by Cohen and Heine, is the condition that the pseudoorbital should minimize the expectation value of the kinetic energy operator, i.e., it should be the "smoothest" pseudoorbital. In this work this condition was applied to determine the pseudo-orbital $\psi$, i.e., the pseudopotential coefficients $\alpha_{i}$.

The valence kinetic energy $T$ is given by the expectation value of the kinetic energy operator $\hat{t}=-\frac{1}{2} \nabla^{2}$ :

$$
T=\frac{\langle\psi|\hat{t}| \psi\rangle}{\langle\psi \mid \psi\rangle} .
$$

The minimization of $T$ leads to

$$
\langle\delta \psi|\hat{t}-T| \psi\rangle=0
$$

Using Eq. (5) we obtain

$$
\left\langle u_{j}|\hat{t}-T| \psi\right\rangle=0 \quad(j=1, \ldots, N) .
$$

From Eqs. (5), (6), and (11) a set of equations can be gained for the coefficients $\alpha_{i}$ :

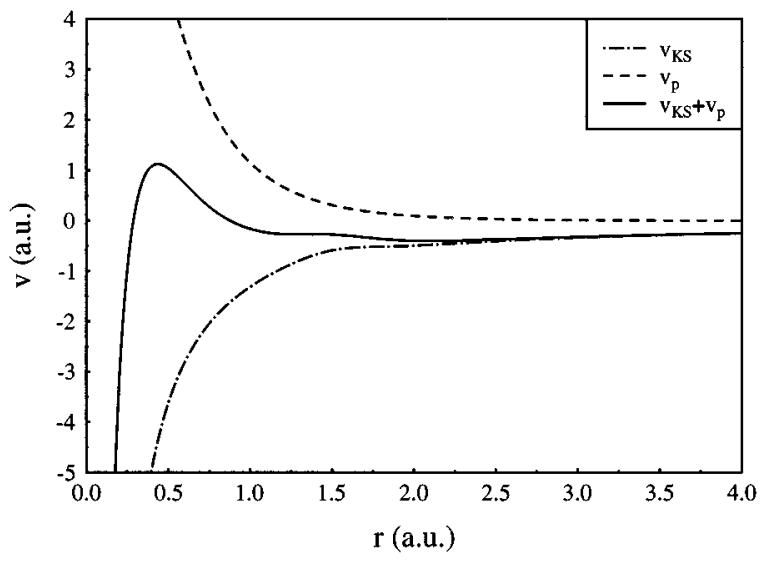

FIG. 4. The Kohn-Sham potential, the pseudopotential, and the modified potential for the $\mathrm{Li}$ atom. 


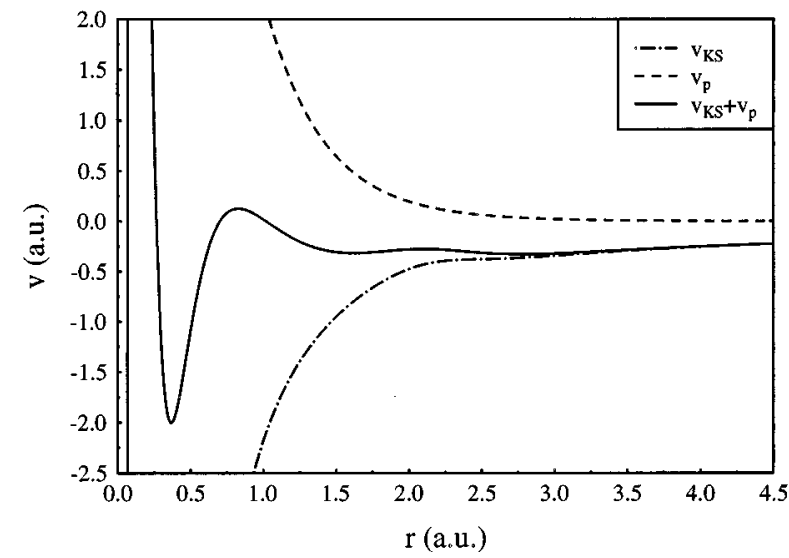

FIG. 5. The Kohn-Sham potential, the pseudopotential, and the modified potential for the $\mathrm{Na}$ atom.

$$
\begin{gathered}
\left(\varepsilon_{j}-T\right) \alpha_{j}-\sum_{i=1}^{N} \alpha_{i}\left\langle u_{j}\left|v_{\mathrm{KS}}\right| u_{i}\right\rangle=\left\langle u_{j}\left|v_{\mathrm{KS}}\right| \varphi\right\rangle \\
(j=1, \ldots, N) .
\end{gathered}
$$

Equation (12) can be solved for $\alpha_{i}$ self-consistently: the first iteration is obtained by inserting simply $\varphi$ instead of $\psi$ in Eq. (9) and then Eq. (12) is a set of linear equations for the coefficients $\alpha_{i}$. Then, using Eqs. (5) and (9), Eq. (12) is solved again to get the second iteration for the coefficients $\alpha_{i}$. The procedure rapidly converges, leading to a selfconsistent solution. Then Eqs. (5) and (8) give the pseudoorbital $\psi$ and the pseudopotential $v_{p}$.

\section{RESULTS AND DISCUSSION}

First, Kohn-Sham potentials have been determined from electron densities. The Hartree-Fock data obtained recently by Bunge et al. [14] have been used to construct the input density. Calculations have been performed for the $\mathrm{Li}, \mathrm{Na}$, and $\mathrm{K}$ atoms.

From the Kohn-Sham orbitals pseudo-orbitals have been calculated by minimizing the kinetic energy. Table I contains

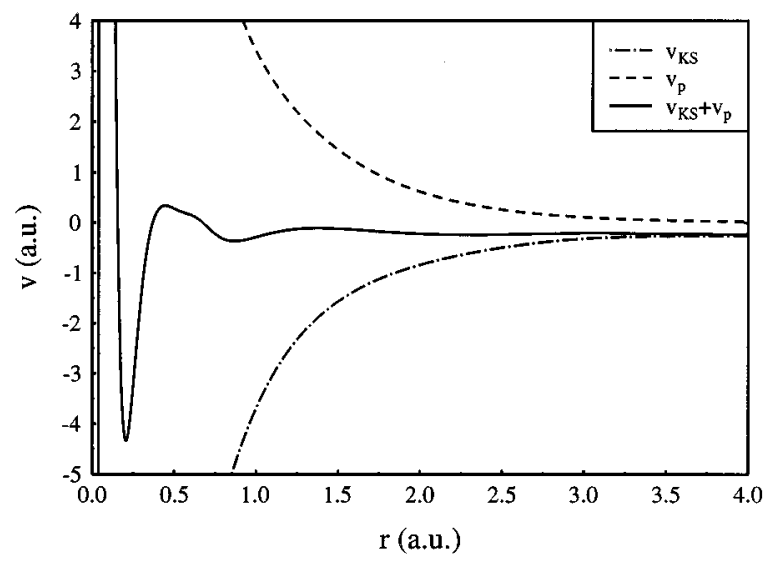

FIG. 6. The Kohn-Sham potential, the pseudopotential, and the modified potential for the $\mathrm{K}$ atom.

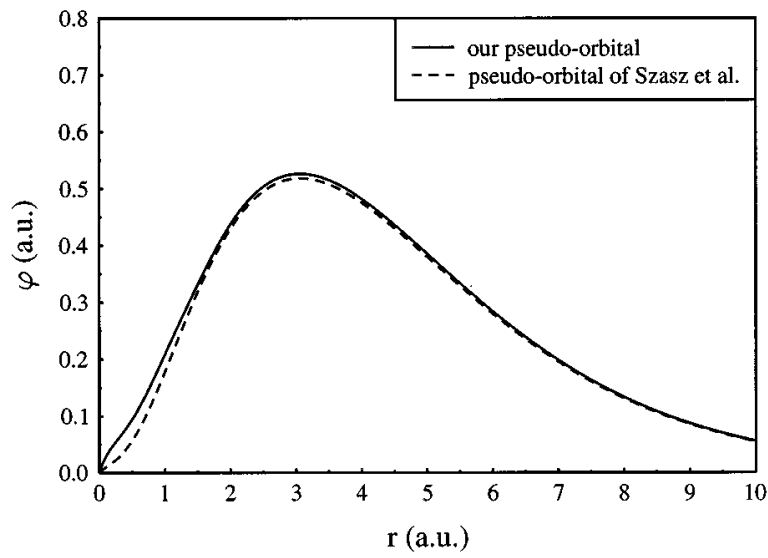

FIG. 7. The pseudo-orbital of the Li atom calculated by ourselves and Szasz and McGinn.

our numerical results for the coefficients $\alpha_{i}$ and Figs. 1-3 show the pseudo-orbitals $\psi$ for these atoms. For comparison they contain the Kohn-Sham orbitals, too. All the orbitals are radial orbitals. As was expected, the pseudo-orbitals are rather smooth.

Then pseudopotentials have been determined from Eq. (8). The Kohn-Sham potentials $v_{\mathrm{KS}}$, the pseudopotentials $v_{p}$, and the modified potentials $v_{\mathrm{KS}}+v_{p}$ are presented in Figs. 4-6. The potential barrier which prevents the valence electron from falling into the core can be clearly seen.

As the input density was the Hartree-Fock density in our calculations, it is very natural to make a comparison with calculations of Szasz and McGinn [15]. They determined pseudopotentials of Phillips-Kleinman type from HartreeFock wave functions using the expression

$$
V_{p}=\sum_{i=1}^{N} \alpha_{i}^{\mathrm{HF}}\left(\varepsilon^{\mathrm{HF}}-\varepsilon_{i}^{\mathrm{HF}}\right) \frac{\varphi_{i}^{\mathrm{HF}}}{\psi^{\mathrm{HF}}},
$$

where $\varphi_{i}^{\mathrm{HF}}$ and $\varepsilon_{i}^{\mathrm{HF}}$ are the Hartree-Fock core orbitals and orbital energies and $\psi^{\mathrm{HF}}$ and $\varepsilon^{\mathrm{HF}}$ are the pseudo-orbital and the valence orbital energies, respectively.

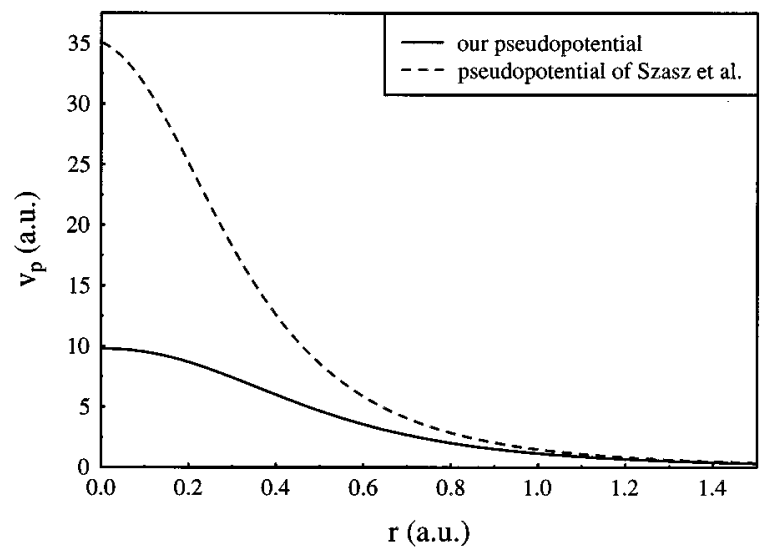

FIG. 8. The pseudopotential of the Li atom calculated by ourselves and Szasz and McGinn. 
TABLE II. The orbital energies (in a.u.) of the $\mathrm{Li}, \mathrm{Na}$, and $\mathrm{K}$ atoms calculated by ourselves and Szasz and McGinn.

\begin{tabular}{|c|c|c|c|c|c|c|c|c|}
\hline \multirow[b]{2}{*}{ Atom } & \multicolumn{4}{|c|}{ Our results } & \multicolumn{4}{|c|}{ Results of Szasz and McGinn } \\
\hline & $1 s$ & $2 s$ & $3 s$ & $4 s$ & $1 s$ & $2 s$ & $3 s$ & $4 s$ \\
\hline $\mathrm{Li}$ & -2.0821 & -0.1963 & & & -2.7924 & -0.1966 & & \\
\hline $\mathrm{Na}$ & -38.0730 & -2.2569 & -0.1820 & & -40.7597 & -3.0737 & -0.1811 & \\
\hline $\mathrm{K}$ & -128.9787 & -13.0587 & -1.3505 & -0.1474 & -133.7524 & -14.7082 & -1.9638 & -0.1467 \\
\hline
\end{tabular}

Figure 7 presents their and our pseudo-orbitals of the $\mathrm{Li}$ atom. These curves are quite close to each other. We have obtained similar results for the other two atoms. One can easily understand this as there is only a small difference between these close-to-exchange-only density-functional and the Hartree-Fock one-electron orbitals [5]. The main cause of the difference between the pseudo-orbitals $\psi$ and $\psi^{\mathrm{HF}}$, as can be seen from Eq. (5), lies in the difference of the coefficients $\alpha_{i}$. Table I shows these coefficients. The differences between the pseudopotential and modified potential determined here and the ones calculated by Szasz and McGinn [15] are quite significant, as can be seen in Fig. 8 for the $\mathrm{Li}$ atom. Comparing Eqs. (8) and (13) we can observe that the reason for this difference is the deviation of the one-electron eigenvalues. As Table II demonstrates, the highest one-electron orbitals are the same but all the others differ considerably $[5,16]$. Here the Hartree-Fock energies of the core orbitals are the one-electron energies of the ions $\mathrm{Li}^{+}$,
$\mathrm{Na}^{+}$, and $\mathrm{K}^{+}$because Szasz and McGinn used them to construct the pseudopotentials.

In conclusion, we can state that a method is worked out to determine pseudopotential in the density-functional theory. This method makes it possible to determine an exact pseudopotential if the exact electron density is available. If, however, the input density is the Hartree-Fock density, a pseudopotential very close to the exact exchange-only one can be obtained.

\section{ACKNOWLEDGMENTS}

This publication is based on work sponsored by the Hungarian-U.S. Science and Technology Joint Fund in cooperation with the National Science Foundation and the Hungarian Academy of Sciences under Project No. 146/91. This work has also been supported by OTKA Grants No. T16623 and No. F16621.
[1] For a review see, e.g., M. C. Payne, M. P. Teter, D. C. Allan, T. A. Arias, and J. D. Joannopoulos, Rev. Mod. Phys. 64, 1045 (1992).

[2] C. O. Almbladh and A. C. Pedroza, Phys. Rev. A 29, 2322 (1994).

[3] F. Aryasetiawan and M. J. Stott, Phys. Rev. B 38, 2974 (1988).

[4] J. Chen and M. J. Stott, Phys. Rev. A 44, 2816 (1991).

[5] Á. Nagy, J. Phys. B 26, 43 (1993); Philos. Mag. B 69, 779 (1994).

[6] Q. Zhao and R. G. Parr, J. Chem. Phys. 98, 543 (1993).

[7] R. G. Parr, Philos. Mag. B 69, 737 (1994).

[8] J. Chen, R. O. Esquivel, and M. J. Stott, Philos. Mag. B 69, 1001 (1994).
[9] A. Görling, Phys. Rev. A 46, 3753 (1992).

[10] J. C. Phillips and L. Kleinman, Phys. Rev. 116, 287 (1959); 118, 1153 (1960).

[11] A. Görling and M. Ernzerhof, Phys. Rev. A 51, 4501 (1995).

[12] A. Holas and N. H. March, in Density Functional Theory, edited by R. Nalewajski, Topics in Current Chemistry Vol. 180 (Springer-Verlag, Heidelberg, 1996).

[13] M. H. Cohen and V. Heine, Phys. Rev. 122, 1821 (1961).

[14] C. F. Bunge, J. A. Barrientos, and A. V. Bunge, At. Data Nucl. Data Tables 53, 114 (1993).

[15] L. Szasz and G. McGinn, J. Chem. Phys. 47, 3495 (1967).

[16] Á. Nagy and N. H. March, Chem. Phys. 153, 141 (1991). 